\title{
The Enlightenment of Folk Paper-cut Art to Visual Communication Design
}

\author{
Liu Chunhua
}

Hunan University of Commerce

hunter2011@foxmail.com

Keywords: Paper-cut art; Decoration; Image; Visual communication

\begin{abstract}
Paper-cut art is a distinctive folk art of Chinese nation, possessing profound and rich national cultural connotation, long historical traditions as well as high artistic values. Folk Paper-cut is an important folk decorative artwork of China, whose special cultural breath and humanism reflects Chinese people's active aesthetic viewpoints and pursuits of beautiful yearnings. Contemporary Paper-cut inherits Chinese nation's local smells and artistic characteristics. This paper discusses four characteristics of paper cut, which are decoration, imagery, conciseness and planarity, thereby explaining the reference and enlightenment of folk Paper-cut to visual communication design.
\end{abstract}

\section{Introduction}

Themes of Paper-cut works reflect harmony and kindness, which symbolizes good luck and happiness. Production methods of Paper-cut are not only skillful and simple, but also have stable artistic forms, presenting different artistic styles and simple and strong artistic charms. Paper-cut combines practicability and decoration effectively, presenting the rich and colorful folk life of Chinese people and satisfying the public's demands for aesthetics.

Functions of visual communication design are to communication the intended information in the first time. Different from other environment design and product design with utility functions, it transmits information by visual symbols rather than language. In the meantime, visual communication is also the process that transforms concepts and ideology into visual symbols. Art and culture play leading and core status in works. They are not only the most stable constituents in the design, but also the essence of design. Therefore, only by knowing traditional art and traditional culture very well and combining it with design philosophy effectively can we create works with excellent form and content. As a representative of Chinese traditional folk culture, Paper-cut art can bring artistic reference and profound enlightenment to visual communication design.

\section{Decoration of Paper-cut art}

Chinese folk Paper-cut art has long standing history. Since it has strong decoration, the production methods are very simple, the vivid models are simple and honest and it has profound local folk culture, it becomes very popular among people and is widely applied in vast people's life.

Paper-cut works are mainly used for putting up and hanging, the purpose of which is to decorate rooms and beautify the environment. Decoration of Paper-cut art is mainly realized by penetration, combination and comparison of lines. In order to satisfy the decoration requirements of beautification and practicability, transformed and exaggerated artistic methods are usually methods for Paper-cut works to express the content. Transformation and exaggeration in Paper-cut works are random and bold and are not restricted by other images or influenced by Western art, thereby creating unique, unreal and romantic art forms and strengthening interest and decoration. Take the Paper-cut of cat eating fish for example. In the work, the plate is cut as plane, which cannot be accepted by people who received professional artistic knowledge. Since according to principles, the plate should be cambered. However, folk paper-cut artists have their own theories, "plates should be plane, or else how could it be put on the table?” Models of the Paper-cut works are brave enough to break through natural restrictions and they try to express their viewpoints through simple forms. In 
folk Paper-cut art, we will always find a lot of lively, interspersing, horizontal and vertical lines, which are not to show the dynamic condition or volume or the structure of the pattern. However, these curved, straight, long and short lines not only increase the rendering of the artistic works, but also enrich the connotation of the artistic works. This processing method embodies the decoration of the works sufficiently. The emptiness of the line and vague decoration have brought more aesthetic significance and spiritual ideas to the works, aesthetic pleasure and subjective reverie to appreciators so that they can understand themes of the works through details.

A piece of excellent Paper-cut work is to present harmonious, opening and closing, slow and fast, hard and soft, rhythm, movement, false or true, density and other forms through the thick and thin, curved and straight, long and short lines, presenting the uncomplicated and simple images of Paper-cut art to people. No matter the lines are curved or straight, long or short, light or dark, simple, smooth, clumsy, fine and smooth or extensive, they contain multiple elements such as texture, space, light and hue and so on, presenting philosophical concept of Chinese ancient yin-yang theory.

\section{Imagery of Paper-cut art}

Image is a kind of conceptual work and is a process that people reflect their own thoughts and emotions as well as objective realties. "Image" means people's mind as well as the reflection of the objective work in subjective thought; it is the presentation of image of the objective world in visual thinking. In Chinese aesthetic category, image means concept, thought and objective things, and it is existence that surpasses subjective awareness. Image possesses unity of opposites of objective things and subjective emotions. It can combine emotions with feelings organically. Here, it means ways of thinking and ideology, which is represented in two aspects, one is word concept, and the other is figure. All in all, image means expressing ideas with images. It is the product of visual effects and thinking, and is expression form of emotions and concepts. From the perspective of semiology, images are symbols that realize heart communication with expressions of objects and forms. Susanne K.Langer once said, "Symbols present their meaning with the simplest forms", which is one of the important reasons that we grasp it.

Models of Chinese folk Paper-cut works contain Chinese traditional thoughts and aesthetic viewpoints, which can be sublimed to inside information and connation of modern design. Folk Paper-cut art puts emphasis on auspicious patterns and is presented with material forms. When people see models of the paper cut, they would think of the beautiful implied meanings. This expression method can integrate interest, meaning and emotions perfectly, expanding aftertaste space of the public and giving rise to resonance. This expression method with Eastern characteristics shows the unique aesthetic viewpoints and complexes of the East. The profound meaning of Chinese folk Paper-cut has the same essence with modern design, which integrates Paper-cut art in plane design and can enhance the profound meaning and connotation of the works.

Pattern imagery. Expression methods of folk Paper-cut art takes the unique aesthetic viewpoints, psychological structure and cultural background as basis, and it utilizes homophonic, token and association and other special ways to materialize auspicious meaning so as to arouse people's psychological resonance for emotional expression. For example, Cheng Jianli's Paper-cut work from Fuyang, Anhui. His Paper-cut works have abundant imageries, simple and honest characters' expressions as well as straightforward emotions. The character shapes are simple and liberal without sticking to trifles. The exaggeration methods are very distinctive. His works have direct imagery, which can reflect people's yearning, longing and pursuit for beautiful life.

From the ancient times, people pray to be happy, prosperous, lively, healthy and peaceful. Therefore, works with this profound meaning are generalized during development of Paper-cut art, thereby becoming fixed auspicious model of Chinese ancient Paper-cut work. Imagery of Paper-cut art patterns present its unique aesthetic characteristics and artistic charms.

Color imagery. In folk Paper-cut art, dyeing and multicolor artistic expressions often emerge, which have strong visual impacts and stronger color contrast. However, colorful Paper-cut is not the mainstream representation forms of Paper-cut art. Basically, Paper-cut works adopt single red, on 
the one hand, it is to show the simplicity of the works; on the other hand, it is to show the primitive worship of China to red as well as the symbolic meaning of red. Chinese people have special complexes to red. It is not only common and traditional color of China, but also a color that is often used in Chinese artistic works. For example, the color of Beijing Olympic logo, the color of the torch as well as the color of the walls of the Imperial Palace. In Chinese people's eyes, red means prosperity, best wishes and auspicious omen as well as awe and praises to life.

\section{Simplicity of Paper-cut art}

Any artistic forms need to satisfy appreciators' visual physiology and psychological needs, and it should guarantee effective, accurate and rapid transmission of visual information. Therefore, simplicity has become an important way to satisfy visual psychology. From the perspective of artistic representation form, simplicity is to highlight themes of the work, delete irrelevant details and show the authors' creation intention and connotation of the works. There are some similarities between simplifying artistic forms and extracting languages. A piece of simple work is just the same as concise language. Only through simple works can people be clear about the themes at a glance and can the work transmit designers' intention effectively.

Folk Paper-cut art not only has simple instruments such as nicking tools and scissors, but also simple materials, which is ticker tape. The expression techniques are Yin cutting method and Yang cutting method. The Paper-cut works also show the lucky implied meaning simply so that people can understand the author's psychological activity and themes of the work at first glance. Applying simplicity of Paper-cut art to modern design can also provide different enlightenment to designers.

\section{Planarity of Paper-cut art}

Folk Paper-cut art works are mainly used for hanging and pasting. Therefore, Paper-cut materials are normally paper of various colors with red as the main color. Production instruments mainly include nicking tool and scissors. It does not have multiple gradation changes as drawing and engraving art. It can only present two gradation effects, which are red and white. Applying characteristics of virtuality and reality combination of Paper-cut art to modern design and strengthening the interaction of virtuality and reality can enhance artistic expressive force of design. Modern design not only pays attention to reality, but also the implementation of virtuality and reality. Modeling of Paper-cut art can bring more ideas and inspirations to design. If applied appropriately, it may bring unexpected effects.

Positive and negative shape is the basis of folk Paper-cut art. To study it must pay attention to Chinese traditional philosophical thought_-Yin and Yang theory. Yin and Yang theory in Paper-cut art is not only represented in outside expression, but also in inner culture. Models of Paper-cut art takes traditional cultures such as ideology, consciousness and beliefs as basis and are inherited and extended through Paper-cut art.

Expression techniques of folk Paper-cut art are realized by Yin cutting method, Yang cutting method and Yin \& Yang mixed cutting method. The above three creation methods are exactly the same with sealing cutting in Chinese traditional seal cutting art, and the differences lie in bearing things and instruments. Yin cutting method means retaining useful patterns and figures and deleting useless ones. Paper-cut works by Yin cutting method are usually dignified and simple with strong visual effects. While signs of ICBC and ABC are master works by Yang cutting method. Hollowing method means combining Yin cutting method with Yang cutting method. It means excavating useless patterns and figures and retaining parts with openwork carving. Works of this style are normally elegant and comely. Yin \& Yang mixed cutting method is effective combination of Yin cutting method and Yang cutting method with complementation of Yin and Yang. Both Yin and Yang have their unique meanings. In the meantime, they relate to each other and form an integrate Paper-cut work. 


\section{Conclusion}

Folk traditional Paper-cut art has a long history and is an art form created and invented in production and life by Chinese working people. In the meantime, it is one of the major forms transmitted by Chinese folk artistic cultures and inherits Chinese folk art characteristics. Paper-cut works have harmonious and beautiful patterns and the materials are proper and extensive. Its artistic forms satisfy the public's demands sufficiently. The forms are not only beautiful and vivid but also have various expression styles. Design of Paper-cut art represents multiple expression forms in modern design and has characteristics of modern art, which can give us reference and enlightenment in visual communication design.

\section{Acknowledgements}

This paper is Hunan Province Philosophy and Social Science Fund Program Applied Research of Hunan Folk Fine Arts in Modern Design of Print Ads

Project Number: 11YBA188

\section{References}

[1] L.Z. Xing. Enlightenment of Folk Paper-cut Art to Visual Communication Design. Journal of Liaocheng University (Social Science Edition),2012,09(2).

[2] M. Zhang. On Paper-cut Creation of Portrait_— Taking the Self-created Men of Undiscovered Talents Come Out in Succession as an Exmaple. Qun Wwen Tian Di,2012,12(8).

[3] D.M. Jiang. On Formal Beauty of Chinese Folk Paper-cut Art.Heilongjiang Shi Zhi, 2014,33(15).

[4] Y. Zhu. A Big World in a Pair of Small Scissors-On Folk Paper-cut Art. Art Fashion (Late of the month Edition),2014,26(2).

[5] Z. Hou. On Nanjing Folk Paper-cut Art. Literature and Art Life. Literature and Art Theory, 2015,11(3). 